2. R. M. Smullyan, Theory of formal systems, Annals of Mathematics Studies No. 47, Princeton Univ. Press, Princeton, N. J., 1961.

3. S. C. Kleene, Introduction to metamathematics, Van Nostrand, New York, 1952.

4. J. C. E. Dekker, A theorem of hypersimple sets, Proc. Amer. Math. Soc. 5 (1954), 791-796.

YESHIVA UNIVERSITY

\title{
ENTROPY FOR NONINVERTIBLE TRANSFORMATIONS
}

\section{JOHN D. FERGUSON ${ }^{1}$}

1. Introduction. In 1959, Sinai (see [1]) gave an improved version of the definition of entropy for a measure-preserving transformation on a probability space. Included in the same paper was a theorem which made possible the computation of the entropy of certain invertible measure-preserving transformations. In this paper we prove a theorem, similar to that of Sinai, for measure-preserving transformations which are not necessarily invertible.

2. Preliminaries. Let $(X, S, \mu)$ be a probability space, and $T$ a measure-preserving transformation on $X$. If $\boldsymbol{A}$ and $\boldsymbol{C}$ are subfields of $\boldsymbol{S}$ with $\boldsymbol{A}$ finite, then the "entropy" and "conditional entropy" of $\boldsymbol{A}$, denoted $\bar{H}(\boldsymbol{A})$ and $\bar{H}(\boldsymbol{A} / \boldsymbol{C})$, respectively, are defined in Halmos [2]. Using these concepts, the entropy of $T$ is defined as follows. Let $\boldsymbol{A}$ be a finite subfield of $S$, then $\bigvee_{j=0}^{m} T^{-i} A$ is a finite subfield of $S$, and it follows from a theorem in information theory that

$$
h(T, \boldsymbol{A})=\lim _{m \rightarrow \infty}(1 /(m+1)) \bar{H}\left(\bigvee_{j=0}^{m} T^{-j} \boldsymbol{A}\right)
$$

exists. Then the entropy of $T$ is $h^{*}(T)$, where

$$
h^{*}(T)=\sup \{h(T, \boldsymbol{A}) \mid \boldsymbol{A} \text { a finite subfield of } \boldsymbol{S}\} .
$$

Sinai's essential idea was to avoid taking the supremum by exhibiting a finite subfield $\boldsymbol{A}$ of $\boldsymbol{S}$ for which $h(T, \boldsymbol{A})$ gave the supremum, but his proof depended on the invertibility of $T$. The theorem proved below replaces the supremum over all finite subfields $\boldsymbol{A}$ by a supre-

Received by the editors February 2, 1963 and, in revised form, August 1, 1963.

${ }^{1}$ Some of the contents of this paper were included in the author's doctoral dissertation, under the direction of Professor G. A. Hedlund, at Yale University, where the author held a National Science Foundation Graduate Fellowship. 
mum over a countable collection of subfields, and allows noninvertible $T$. We require the following results, which may be found in Halmos [2].

Let $A, B, C, D$ be subfields of $S$ with $A$ and $B$ finite, then

(1) $\bar{H}(A)=-\sum_{A} \mu(A) \log \mu(A)$, where the summation is over all the atoms $A$ of $A$.

(2) $\bar{H}(A \bigvee B)=\bar{H}(B)+\bar{H}(A / B)$.

(3) If $A \subseteq B$, then $\bar{H}(A) \leqq \bar{H}(B)$.

(4) If $A \subseteq C$, then $\bar{H}(\boldsymbol{A} / \boldsymbol{C})=0$.

(5) $\bar{H}\left(T^{-1} \boldsymbol{A} / T^{-1} \boldsymbol{C}\right)=\bar{H}(\boldsymbol{A} / \boldsymbol{C})$.

(6) $\bar{H}(A \vee B / C) \leqq \bar{H}(A / C)+\bar{H}(B / C)$.

(7) If $C \subseteq D$, then $\bar{H}(A / D) \leqq \bar{H}(\boldsymbol{A} / \boldsymbol{C})$.

(8) If $\boldsymbol{C}_{1} \subseteq \boldsymbol{C}_{2} \subseteq \cdots$ are subfields of $\boldsymbol{S}$ then

$$
\lim _{n \rightarrow \infty} \bar{H}\left(\boldsymbol{A} / \boldsymbol{C}_{n}\right)=\bar{H}\left(\boldsymbol{A} / \bigvee_{n=1}^{\infty} \boldsymbol{C}_{n}\right) .
$$

\section{Computing entropy.}

THEOREM. Let $T$ be a measure-preserving transformation on $X$. Suppose there exists a sequence $\left\{\boldsymbol{A}_{n}\right\}, n=1,2, \cdots$, of finite subfields of $\boldsymbol{S}$, such that, if $B_{n}$ denotes $\bigvee_{j=0}^{\infty} T^{-i} A_{n}$, then

(a) $B_{1} \subseteq B_{2} \subseteq \cdots$, and

(b) $S=\bigvee_{n=1}^{\infty} B_{n}$.

Then $h^{*}(T)=\lim \sup h\left(T, A_{n}\right)$.

Proof. Let $B$ be a finite subfield of $S$. Then, for $m, k \geqq 0$, and $n \geqq 1$, it follows that

$$
\begin{aligned}
\bar{H}\left(\bigvee_{i=0}^{k} T^{-i} B\right) & \leqq \bar{H}\left(\bigvee_{i=0}^{k} T^{-i} B \bigvee \bigvee_{j=0}^{m+k} T^{-j} A\right) \\
& =\bar{H}\left(\bigvee_{j=0}^{m+k} T^{-j} A_{n}\right)+\bar{H}\left(\bigvee_{i=0}^{k} T^{-i} B / \bigvee_{j=0}^{m+k} T^{-j} A_{n}\right),
\end{aligned}
$$

by (3) and (2). Now the second term in the sum above is dominated by

$$
\sum_{i=0}^{k} \bar{H}\left(T^{-i} B / \bigvee_{j=0}^{m+k} T^{-j} A_{n}\right),
$$

according to (6), and this is less than or equal to

$$
\sum_{i=0}^{k} \bar{H}\left(T^{-i} B / \bigvee_{j=i}^{m+i} T^{-j} A_{n}\right)
$$


according to (7). Now for any pair of subfields $A$ and $B$ of $S, T^{-1}(A \vee B)$ $=T^{-1} A \bigvee T^{-1} B$, therefore

Thus,

$$
\bigvee_{j=i}^{m+i} T^{-j} A_{n}=T^{-i}\left(\bigvee_{j=0}^{m} T^{-j} A_{n}\right)
$$

by (5). Hence

$$
\begin{aligned}
\bar{H}\left(\bigvee_{i=0}^{k} T^{-i} B / \bigvee_{j=0}^{m+k} T^{-j} A_{n}\right) & \leqq \sum_{i=0}^{k} \bar{H}\left(T^{-i} B / T^{-i} \bigvee_{j=0}^{m} T^{-j} A_{n}\right) \\
& =\sum_{i=0}^{k} \bar{H}\left(B / \bigvee_{j=0}^{m} T^{-j} A_{n}\right) \\
& =(k+1) \bar{H}\left(B / \bigvee_{j=0}^{m} T^{-j} A_{n}\right),
\end{aligned}
$$

$$
\begin{aligned}
\frac{1}{k+1} \bar{H}\left(\bigvee_{i=0}^{k} T^{-i} B\right) \leqq & \frac{m+k}{1+k} \cdot \frac{1}{m+k} \cdot \bar{H}\left(\bigvee_{j=0}^{m+k} T^{-j} A_{n}\right) \\
& +\bar{H}\left(B / \bigvee_{j=0}^{m} T^{-j} A_{n}\right) .
\end{aligned}
$$

Hence, letting $k \rightarrow \infty$,

$$
h(T, B) \leqq h\left(T, A_{n}\right)+\bar{H}\left(B / \bigvee_{j=0}^{m} T^{-j} A_{n}\right),
$$

and, letting $m \rightarrow \infty$,

$$
h(T, B) \leqq h\left(T, A_{n}\right)+\bar{H}\left(B / B_{n}\right), \text { by }(8) .
$$

Finally, letting $n \rightarrow \infty$,

$$
\begin{aligned}
h(T, B) & \leqq \lim \sup h\left(T, A_{n}\right)+\bar{H}\left(B / \bigvee_{k=1}^{\infty} B_{k}\right) \\
& =\lim \sup h\left(T, A_{n}\right), \text { by (8) and (4). }
\end{aligned}
$$

It now follows that $h^{*}(T)=\sup h\left(T, \boldsymbol{A}_{n}\right)=\lim \sup h\left(T, \boldsymbol{A}_{n}\right)$. This completes the proof of the theorem.

4. An example. Let $S$ denote the set of all infinite sequences of zeros and ones, with uniform product measure (see [3, p. 5]), and let $n$ be an integer greater than one. If $f\left(x_{1}, x_{2}, \cdots, x_{n}\right)$ is a polynomial over GF2, then $f$ induces a mapping, $f_{\infty}$, of $S$ into $S$, defined as follows: if $y=\left\{y_{m}\right\}, m=0, \pm 1, \pm 2, \cdots$, then $f_{\infty}(y)=z$ where $z=\left\{z_{m}\right\}$, and 
$z_{m}=f\left(y_{m}, y_{m+1}, \cdots, y_{m+n-1}\right), m=0, \pm 1, \pm 2, \cdots$. Concerning $f_{\infty}$, the following statements are valid:

(a) $f_{\infty}$ is a measurable transformation of $S$ into $S$.

(b) $f_{\infty}$ is measure-preserving iff $f_{\infty}$ is onto.

(c) If $f\left(x_{1}, x_{2}, \cdots, x_{n}\right)=x_{1}+g\left(x_{2}, \cdots, x_{n-1}\right)+x_{n}$, where $g$ is a polynomial over GF2, then:

(i) $f_{\infty}$ is precisely $2^{n-1}$ to 1 , and onto;

(ii) $f_{\infty}$ is ergodic and strongly mixing;

(iii) the entropy of $f_{\infty}$ is $(n-1) \log 2$.

The proof of (c) (iii) may be accomplished by using the theorem proved above (see [4]); however, since $f_{\infty}$ is not invertible, Sinai's theorem cannot be used.

\section{REFERENCES}

1. Y. Sinai, On the concept of entropy for a dynamical system, Dokl. Akad. Nauk SSSR 124 (1959), 768-771.

2. P. R. Halmos, Entropy in ergodic theory, Lecture Notes, University of Chicago, Chicago, Illinois, 1959.

3. - Lectures on ergodic theory, Chelsea, New York, 1956.

4. J. D. Ferguson, Some properties of mappings on sequence spaces, Dissertation, Yale University, New Haven, Connecticut, 1962.

Institute for Defense Analyses 